\title{
Functional Dissociation of $\theta$ Oscillations in the Frontal and Visual Cortices and Their Long-Range Network during Sustained Attention
}

\author{
Hio-Been Han, ${ }^{1,2^{*}}$ Ka Eun Lee, ${ }^{2,3^{*}}$ and Jee Hyun $\mathrm{Choi}^{2,4}$
}

https://doi.org/10.1523/ENEURO.0248-19.2019

\begin{abstract}
${ }^{1}$ Program of Brain and Cognitive Engineering, Korea Advanced Institute of Science and Technology, Daejeon 34141, Republic of Korea, ${ }^{2}$ Center for Neuroscience, Korea Institute of Science and Technology, Seoul 02792, Republic of Korea, ${ }^{3}$ College of Liberal Studies, Seoul National University, Seoul 08826, Republic of Korea, and ${ }^{4}$ Department of Neuroscience, University of Science and Technology, Daejeon 34133, Republic of Korea
\end{abstract}

\begin{abstract}
$\theta$-Band $(4-12 \mathrm{~Hz})$ activities in the frontal cortex have been thought to be a key mechanism of sustained attention and goal-related behaviors, forming a phase-coherent network with task-related sensory cortices for integrated neuronal ensembles. However, recent visual task studies found that selective attention attenuates stimulus-related $\theta$ power in the visual cortex, suggesting a functional dissociation of cortical $\theta$ oscillations. To investigate this contradictory behavior of cortical $\theta$, a visual Go/No-Go task was performed with electroencephalogram (EEG) recording in C57BL/6J mice. During the No-Go period, transient $\theta$ oscillations were observed in both the frontal and visual cortices, but $\theta$ oscillations of the two areas were prominent in different trial epochs. By separating trial epochs based on subjects' short-term performance, we found that frontal $\theta$ was prominent in good-performance epochs, while visual $\theta$ was prominent in badperformance epochs, exhibiting a functional dissociation of cortical $\theta$ rhythms. Furthermore, the two $\theta$ rhythms also showed a heterogeneous pattern of phase-amplitude coupling with fast oscillations, reflecting their distinct architecture in underlying neuronal circuitry. Interestingly, in good-performance epochs, where visual $\theta$ was relatively weak, stronger fronto-visual long-range synchrony and shorter posterior-to-anterior temporal delay were found. These findings highlight a previously overlooked aspect of long-range synchrony between distinct oscillatory entities in the cerebral cortex and provide empirical evidence of a functional dissociation of cortical $\theta$ rhythms.
\end{abstract}

Key words: EEG; frontal $\theta$; oscillations; sustained attention; $\theta$; visual $\theta$

\section{Significance Statement}

Previous literature emphasized the pro-cognitive role of coherent oscillatory networks between distal brain regions, such as the fronto-visual $\theta$ synchrony. However, such a conceptual framework has been challenged as recent findings revealed distinct behavioral correlates of $\theta$ oscillations found in different cortical regions, especially in the frontal and visual cortices. Here, we show that frontal and visual $\theta$ represent distinct cortical processes and that the functional connectivity between them increases during sustained attention, especially when one of the two $\theta$ rhythms is relatively suppressed. The data presented here highlight a novel aspect of neural long-range synchrony between distinct cortical oscillators with distinct functional significance in task performance. 


\section{Introduction}

In the mammalian brain, oscillatory networks in the lower frequency bands $(<12 \mathrm{~Hz})$ such as the $\theta$ band $(4-12$ $\mathrm{Hz}$ ) are often thought to be a key mechanism of brainwide interaction between distal regions (von Stein and Sarnthein, 2000). In particular, increased $\theta$ rhythmic activity of the frontal cortex is a hallmark of improved task performance and sustained attention, known to reflect the level of cognitive demand of the given task (Cavanagh and Frank, 2014; Clayton et al., 2015). In addition, $\theta$ oscillations of the frontal cortex have been known to form a phase-coherent network with task-related brain regions, including the sensory cortices (Benchenane et al., 2011). The emergence of a $\theta$-oscillation network reflects active neuronal processing for cognitive functions such as sustained attention (Clayton et al., 2015), cognitive control (Cavanagh and Frank, 2014), and working memory (Sauseng et al., 2010). For instance, Liebe et al. (2012) reported local-field potentials and unit activities that were synchronous across the prefrontal and visual areas in the $\theta$ band during visual working memory tasks.

On the other hand, recent findings suggested that $\theta$ activities of the visual area are distinct from those of the frontal cortex and that visual $\theta$ is also associated with attention but in an opposite manner: stimulus-evoked $\theta$ power decreased with increased attention. For instance, Spyropoulos et al. (2018) recently reported that $\theta$ oscillations in the visual cortex elicited by visual stimuli decreased in amplitude when visual attention was directed. In addition, another recent study found arousal-reduced $\theta$ power in the high-order visual area (i.e., posterior parietal cortex) of the ferret brain (Stitt et al., 2018) despite its enhanced long-range $\theta$ synchrony with the prefrontal cortex during sustained attention reported by an earlier study (Sellers et al., 2016). In fact, a pioneering neuro-feedback study in the 1970 s reported similar results of an association between occipital $\theta$ suppression and improved performance in a visual detection task (Beatty et al., 1974). There have also been several reports of enhanced $\theta$ in the posterior brain region being predictive of inattentiveness such as after sleep deprivation (Vyazovskiy and Tobler, 2005) or an attentional lapse (Mazaheri et al., 2009). Although the term "attention" of each study referred to

Received June 27, 2019; accepted October 28, 2019; First published November 4, 2019.

The authors declare no competing financial interests.

Author contributions: H.-B.H. and J.H.C. designed research; H.-B.H., K.E.L., and J.H.C. performed research; H.-B.H. and K.E.L. analyzed data; H.-B.H., K.E.L., and J.H.C. wrote the paper.

This work was supported by the National Research Council of Science and Technology of Korea for the project Development of Solution for Diagnosis, Treatment, and Care System of Dementia Grant CRC-15-04-KIST, the K-DARPA project (2V0713) and by the National Research Foundation of Korea (NRF) Grant 2017R1A2B3012659.

*H.-B.H. and K.E.L. are first authors.

Correspondence should be addressed to Jee Hyun Choi at jeechoi@kist.re.kr.

https://doi.org/10.1523/ENEURO.0248-19.2019

Copyright (C) 2019 Han et al.

This is an open-access article distributed under the terms of the Creative Commons Attribution 4.0 International license, which permits unrestricted use, distribution and reproduction in any medium provided that the original work is properly attributed. different aspects of attention (e.g., internal or external attention; Chun et al., 2011) on different time scales (e.g., minute or second levels), evidence generally suggests that the employment of attention yields contrary patterns of $\theta$ oscillations in the frontal and visual cortices.

This attentional modulation of frontal and visual $\theta$ in opposite directions poses a question about the functionality of $\theta$ oscillations in different brain regions, especially in terms of their long-range functional connectivity. If cortical $\theta$ oscillations of different regions have different roles with opposing behavioral correlates, how could they form a coherent oscillatory network? A possible explanation of this counterintuitive interaction between cortical oscillators is that the oscillations of one region are suppressed to achieve synchronization with those of the other region. Here, we hypothesize that enhanced frontovisual $\theta$ synchrony can occur during the suppression of frontal or visual $\theta$.

To address this hypothesis, we designed a forcedpaced (i.e., the opposite of self-paced) visual Go/No-Go task for head-fixed mice and analyzed the electroencephalogram (EEG) oscillations of each area. With this design, we aimed to capture the dynamic interaction between frontal and visual $\theta$ within a single protocol. In particular, in No-Go trials, we expected the recruitment of both frontal and visual $\theta$ oscillations, depending on the attentive state of the animal. This is because elaborate sustained attention (i.e., remaining alert while inhibiting a Go response) has been known to elicit control-related $\theta$ in the frontal cortex (Clayton et al., 2015; Sellers et al., 2016), while visual perception without attention has been reported to elicit $\theta$ oscillations in the visual area (Spyropoulos et al., 2018). By tracking short-term behavioral performance within a single experimental session, we investigated state-dependent changes of frontal and visual $\theta$ oscillations and their coupling with higher frequency oscillations (e.g., $\gamma$ oscillations) to confirm their functional dissociation. Then, we characterized the property of functional connectivity between frontal and visual $\theta$ to show how distinct cortical oscillators are coupled to form a coherent long-range network.

\section{Materials and Methods}

All procedures were approved by the Institutional Animal Care and Use Committees at the corresponding author's primary affiliation and complied with $\mathrm{NIH}$ guidelines. Detailed experimental procedures were as follows.

\section{Animals and surgery}

Five male C57BL/6 mice (four to eight weeks old at time of surgery, body weight $\mathrm{M}=26.1 \mathrm{~g}, \mathrm{SD}=4.7 \mathrm{~g}$ ) were used, maintained under a 12/12 h light/dark cycle (lights on at 8 A.M.) in a temperature-controlled and humiditycontrolled environment. Food was available ad libitum, and water was mildly restricted $(1 \mathrm{ml} / \mathrm{d}$, additional water available as reward from task) from two weeks after surgery. Health status of the animals was assessed daily according to a systematic assessment protocol (Guo et al., 2014). All procedures were conducted in compliance with the Institutional Animal Care and Use Commit- 
tee of corresponding author's primary affiliation, conforming to the NIH Guide for Care and Use of Laboratory Animals (NIH Publication No. 86-23, revised 1985).

For stereotaxic surgery, mice were anesthetized with a ketamine and xylazine cocktail (120 and $6 \mathrm{mg} / \mathrm{kg}$, respectively) by intraperitoneal injection and positioned on a stereotaxic apparatus (Model 957, Kopf Instruments). After shaving the head, an incision was made to expose the skull and implant sterilized screw (Asia Bolt) electrodes above the frontal (anterior-posterior, 1.9-2.1 mm; mediallateral, $\pm 2.1 \mathrm{~mm}$ from bregma) and visual cortices (anterior-posterior, $-3.7 \mathrm{~mm}$; medial-lateral, $\pm 3.7 \mathrm{~mm}$ from bregma). For selection of recording sites, we referred to a previous study which targeted the mouse prelimbic area to record $\theta$ oscillations using surface electrodes in vivo (Sauer et al., 2015), and our pilot study using highdensity surface EEG array (Choi et al., 2010) which showed that the selected mouse visual area exhibited most prominent motion-specific oscillatory responses to random-dot motion stimuli. Reference and ground electrodes were implanted on the occipital bone (Paxinos and Franklin, 2004). Dental cement (Vertex Self-Curing, Vertex Dental) was applied over the electrodes for fixation and two polycarbonate nuts (inner diameter $3 \mathrm{~mm}$, Nippon Chemi-Con) were attached to the caudal edges of the cement, for use as anchors of the custom-built headfixation apparatus. After surgery, mice were treated with antibiotics and analgesics.

\section{Stimuli and apparatus}

All experiments were conducted in a lightproof Faraday cage. Mice were restrained in a custom-built acrylic tube (3.5 cm in diameter) and head fixed by the polycarbonate nuts. For a reward, a water drop $(3 \mu \mathrm{l})$ was released through a pressure equalizer tube ( $2 \mu \mathrm{m}$ in diameter) with a programmable syringe pump (Fusion 200, Chemyx). Licking behavior was counted with a custom-built lickometer made of stainless-steel wire (3 $\mu \mathrm{m}$ in diameter) and a data acquisition system (cDAQ-9147, National Instruments). For punishment, a single puff of air was delivered to the animal's cheek (air pressure $=15-20$ psi, duration $=0.2 \mathrm{~s}$ ), supplied with a compressed air tank and controlled by a solenoid valve. A schematic diagram of the experimental setup is shown in Figure $1 A$.

For visual stimuli, a random-dot motion was generated and presented through MATLAB (MathWorks). The dot motion was composed of 100 small dots (full coherence, size $=0.5^{\circ}$ in radius, field size $=60^{\circ}$ in diameter, speed $=$ $42^{\circ} \mathrm{s}$, dot life $=1.67 \pm 0.33 \mathrm{~s}$ from a Gaussian distribution, orientation $=90^{\circ}$ or $180^{\circ}$ ) and presented on a LCD screen (LG E1910PM-SN, 19 inches, $1280 \times 1024$ resolution), located $15 \mathrm{~cm}$ away from the animal's eyes. As for the validity of using random-dot motions in the mouse model, previous studies have shown rodents are able to discriminate the direction of the random-dot motion (Douglas et al., 2006) and exhibit brain oscillatory responses similar to those found in humans (Han et al., 2017).

\section{Training procedure}

The behavioral training procedure was consisted of three stages: (1) habituation (7 d), (2) response shaping $(2-3 d)$, and (3) conditioning ( $2-4 d)$. The habituation stage includes animal handling (10-15 min/d) and provides animals the opportunity to adapt to the head-fixation setup inside the experimental apparatus (30 $\mathrm{min} / \mathrm{d}$ ) without behavioral requirements. The response shaping stage was aimed to teach animals that water can be accessed by licking the tube. During this period, a water reward was given for every lick (lick port sensor time-out $=20 \mathrm{~s}$ ). Once the animal started to robustly lick the tube, they advanced to the conditioning stage. The conditioning stage trained animals to associate a visual stimulus with a water reward. Each trial began with a monotone sound cue $(10 \mathrm{kHz}, 70 \mathrm{~dB}, 100 \mathrm{~ms})$ and a white central fixation cross $\left(\right.$ size $\left.=3^{\circ}\right)$. After 2-3 s of the cue, a rightward moving-dots stimulus (S+, "Go" motion) was presented on the screen, which subsequently turned black for $30 \mathrm{~s}$ for the water-tube licking. Each stimulus did not last longer than $2 \mathrm{~min}$, and 100 trials were given in $1 \mathrm{~d}$. Our pilot study of reaction time (RT), validated through video recording, showed that intentional licking results in a typical RT distribution with a peak near $1 \mathrm{~s}$, whereas spontaneous licking results in a scattered RT distribution over a 2 min time period. Also, we found that licking rates decreased over time, potentially due to thirst satiation. Thus, we considered the conditioning was successfully established when the RT of the first 80 trials reached a typical RT distribution. Overall, the percentage of mice licking in response to the visual stimulus on the last day of the conditioning stage was $\sim 70 \%$.

\section{Go/No-Go task}

After mice learned to discriminate the direction of the random-dot motion on a screen, their task performance was tested by randomly presenting rightward (Go) or downward ("No-Go") motion of random dots that stopped at licking or after $4 \mathrm{~s}$ when licking was not detected. Each mouse performed 10 experimental sessions on successive days. The task required the animals to discriminate the direction of the moving dots (Fig. 1B). Each trial began with a monotone sound cue $(10 \mathrm{kHz}, 70 \mathrm{~dB}, 100 \mathrm{~ms})$ and a white central fixation cross $\left(\right.$ size $\left.=3^{\circ}\right)$. After $2-3$ s of the cue, the stimulus was presented for $4 \mathrm{~s}$ with either a rightward (S+, Go motion) or downward (S-, No-Go motion) motion which was terminated when licking was detected. The direction of the motion of each trial was randomly determined $(50 \%)$ with a restriction that the same stimulus was not presented more than four times in a row. A licking response during the $\mathrm{S}+$, which was counted as a hit, resulted in instant reward. If the mouse did not take the reward within $20 \mathrm{~s}$, the water in the tube was suctioned out. On the other hand, a licking response to the S- was counted as a FA, resulting in instant punishment and a 20-s timeout. Trials without licking responses were counted as a Miss or CR for the S+ or S-, respectively. To guide the animals to react more consciously, rather than reflexively, the response window started $0.5 \mathrm{~s}$ after the onset of motion (Fig. 1B). Intertrial 


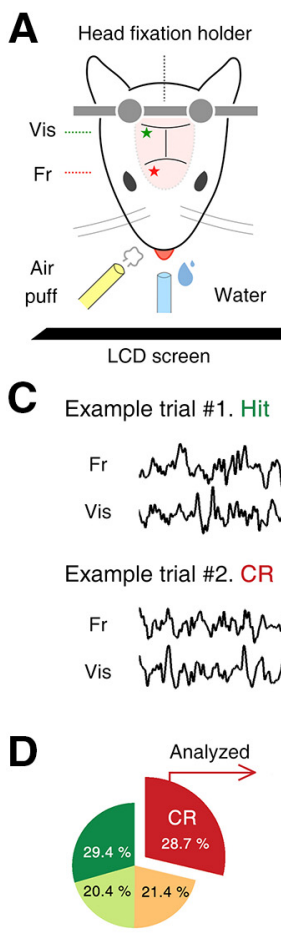

B

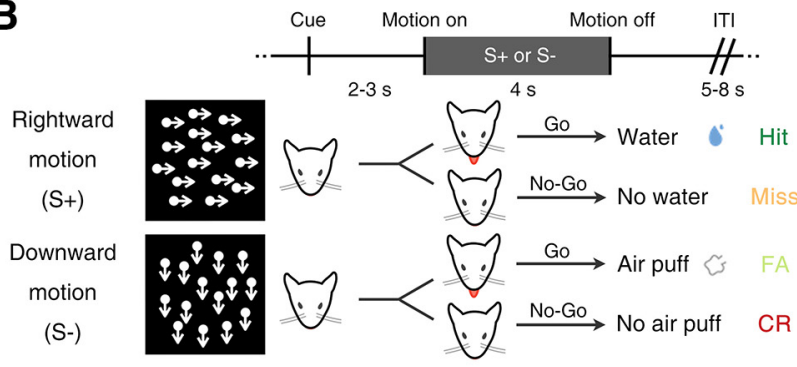

S+ motion Licks. . . ...

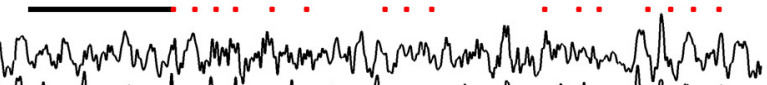

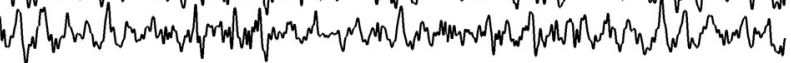

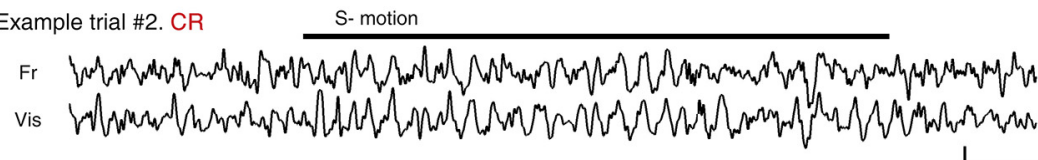

Hit $(n=2,469)$ CR $(n=2,411)$

D

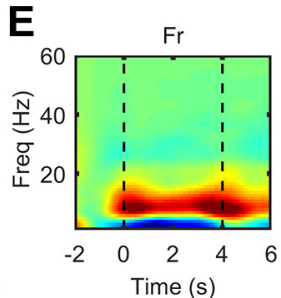

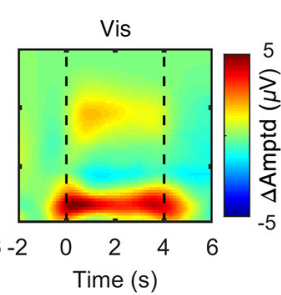

$\mathbf{F}$

Figure 1. $\theta$ oscillations during Go/No-Go task in frontal and visual cortices. A, Schematic illustration of experimental setup. Mice performed Go/No-Go task in custom-built head restrainer. B. Experimental procedures. Task required subject to discriminate direction of random-dot motion (rightward: S+, downward: S-). Go response (i.e., licking) to S+ and S- motion resulted in reward and punishment, respectively. There was no particular reinforcing stimulus following No-Go response (i.e., no licking). The variation of the performance across days and within session was analyzed (Extended Data Fig. 1-1). C, Example EEG data of two trials. Upper, For $\mathrm{S}+$ motion, licking response was counted as "hit," and water reward was followed instantaneously. Motion stimulus was ceased at first lick (RT). Lower, For S- motion, No-Go response was counted as "CR." Scale bars on bottom right indicate $500 \mathrm{~ms}$ and $300 \mu \mathrm{V}$. $D$, Summary of behavioral results. From all subjects (five mice), and all sessions (10 d), data of 8390 trials were collected. In this study, only the trials from $\mathrm{CR}(n=2411)$ were analyzed. $\boldsymbol{E}$, Time-frequency representation of grand-averaged event-related spectral perturbation (ERSP) of each cortical area. In both the frontal and visual cortices, prominent $\theta(4-12 \mathrm{~Hz})$ oscillations were observed during $4 \mathrm{~s}$ of stimulus presentation (No-Go period). The stimulus-locked and response-locked spectrograms in all trials are summarized in Extended Data Figure 1-2. F, Temporal average (0.5-4 s) of $\theta$ oscillations in each cortical area. Numbers denote peak frequency of each channel. FA: false alarm, Fr: frontal cortex, Vis: visual cortex, Amptd: amplitude, Freq: frequency.

intervals (it is; black screen) were 5-8 s long. Any spontaneous licking during the ITI or cue period before the response window reset the trial starting from the ITI.

The number of trials per session was determined by the animal's performance that day (287 trials/d on average). Each session was terminated once the animal stopped licking for 30 successive trials, which we regarded as a lack of motivation for the task, and were excluded from the EEG analysis. We unexpectedly observed an obsessive licking behavior, during which the animal would indiscriminately lick the tube regardless of stimulus type. If animals licked for $>30$ trials in a row, those trials were excluded from EEG analysis. Overall, around $41 \%$ of trials were excluded.

\section{Rating task performance}

The ongoing task performance of mice was assessed by the ratio of correct answers [hit or correct rejection (CR)]. In human and animal studies, it has been known that the performance in cognitive tasks varies over time depending on the behavioral state, for instance due to an attentional lapse (Liebe et al., 2012; Esterman et al., 2013). To investigate the relationship between cortical $\theta$ activities and sustained attention, it was important to infer correctly the behavioral state, as either attentive or inattentive. This is particularly important in Go/No-Go tasks because mind wandering for a short period of time (i.e., attentional lapse) can be counted as a correct No-Go response. To rate each trial according to attentional state, the consistency of performance over time was analyzed. Because the span of attentional state in humans was reported to range between 2 and 7 min (Esterman et al., 2013) or exceed $10 \mathrm{~min}$ (Clayton et al., 2015), we assumed that short-term attentional state in mouse would be similar or shorter than that of human. Then, we assumed the level of attentional state is reflected through performance in the previous trials (in the order of a few minutes). Finally, we identified fluctuations in performance over 10 successive trials by calculating the probability of correct answers $\left(p_{c}\right.$; hit and CR), ranging from 0 (0 correct trials) to 1 (10 
correct trials) with the chance level $0.5 . p_{c}$ of $k$-th trial was defined as follows,

$$
p_{c}(k)=\frac{1}{10} \sum_{n=k-9}^{k} C(n),
$$

where the binary function, $C(n)$, indicates the outcome of the trial as correct (1) or not (0). In our paradigm, 10 trials lasted $\sim 2-5 \mathrm{~min}$. We subsequently classified the trials into attentive state $\left(p_{c}\right.$ larger than the chance level) or inattentive state $\left(p_{c}\right.$ equal to or smaller than the chance level).

\section{EEG data acquisition and preprocessing}

EEG responses were recorded at a 2-kHz sampling rate with microscrew electrodes implanted on the skull above the frontal and visual cortices using Grass 8-17C amplifiers (Grass Technologies, $60-\mathrm{Hz}$ notch filters, gain = $50,000)$ and data acquisition system (cDAQ-9147). After bandpass filtering $(1-200 \mathrm{~Hz})$, the EEG data were processed on a trial-by-trial basis. To eliminate hemispherespecific activities, EEG signals from bilateral electrodes were averaged in the time domain. To minimize the effect of early evoked activities (e.g., event-related potentials), only the EEG activities from 0.5 to $4 \mathrm{~s}$ after motion onset were used for statistical testing of oscillation amplitudes. We computed the mean and two-tailed 99\% confidence intervals of the distribution of the overall amplitude spectrum, and the values lying outside of these confidence intervals were excluded. A time-frequency amplitude spectrogram was obtained by applying fast Fourier transform with a sliding Hanning window (bin size $=512 \mathrm{~ms}$, step size $=100 \mathrm{~ms}$ ) and by taking absolute real parts from the epochs, which resulted in a $1 \mathrm{~Hz}$ frequency resolution. For the time-frequency spectrogram visualization, the amplitudes of the pre-stimulus periods (2-0.5 s before stimulus onset) were averaged and subtracted in a frequencywise manner.

\section{Cross-frequency coupling (CFC) between $\theta$ and $\gamma$ oscillations}

To investigate CFC between $\theta$ and $\gamma$ oscillations, we calculated the modulation index (MI) as described in Tort et al. (2010). Briefly: (1) raw EEG epochs were band passed (phase frequency: 4-12 Hz, amplitude frequencies: $6-200 \mathrm{~Hz}$ with $2.5-\mathrm{Hz}$ step sliding window and $5-\mathrm{Hz}$ width) with 5th order Butterworth filters. (2) The time series of the phase of $\theta$ oscillations denoted as $\phi_{f}(t)$, and the time series of the amplitude of the $\gamma$ oscillations denoted as $A_{f}(t)$, were extracted via Hilbert transform. (3) The phase of the $\theta$ oscillations was divided into $N$ bins from $-\pi$ to $\pi$. In each bin, the average of $A_{f}(t)$ was calculated and then normalized by dividing the sum over the $N$ bins and was denoted as $P_{j}$ for the $j$-th bin (here, $P_{j}$ equals the probability density function of the $\gamma$ amplitude distribution over the $\theta$ phase ranging from 0 to 1). (4) An entropy index, $H$, was calculated by the definition of Shannon entropy, given by

$$
H=-\sum_{j}^{N} P_{j} \log P_{j},
$$

if the $\gamma$ oscillations are independent from $\theta$ phase, $P_{j}$ is $1 / N$; therefore $H=\log (N)$. (5) The statistical deviance of the measured distribution $(P)$ from the uniform distribution was calculated by adopting Kullback-Leibler distance by the following formula,

$$
\mathrm{D}_{K L}=\log (N)-H,
$$

where $D_{K L}$ stands for the Kullback-Leibler distance. (6) Finally, MI was defined with a Z-score of observed entropy as the following formula indicates,

$$
M I=\frac{D_{K L}}{\log (N)} .
$$

In the current study, we set $N$ equal to 30 (i.e., bin size $=12^{\circ}$ ).

\section{Generalized linear model (GLM) analysis of CFC}

Single-trial GLM analyses were performed to investigate the increased level of $\theta-\gamma$ coupling during attentive state is modulated by either $\theta$ amplitude or attentive state, or both. The model was composed of the following equation,

$$
y=\beta_{0}+\beta_{1} x_{1}+\beta_{2} x_{2}+\beta_{3} x_{1} \cdot x_{2}+\epsilon,
$$

where $\mathrm{y}, \mathrm{x}_{1}, \mathrm{x}_{2}$, and $\epsilon$ represent $\mathrm{MI}, \theta$ amplitude, attentional state (inattentive $=0$, attentive $=1$ ), and error term, respectively. The term $x_{1} \cdot x_{2}$ represents a two-way interaction between $\theta$ amplitude and attentional state. GLM fitting was done by a MATLAB built-in function (fitlm.m).

\section{Phase synchrony analysis of $\theta$ activities}

To quantify the interaction between $\theta$ oscillations in the frontal and visual areas, phase synchrony was estimated by joint phase histograms and by calculating phase locking values (PLVs; Lachaux et al., 1999). To obtain joint phase histograms, a MATLAB built-in function for twodimensional histogram (hist2.m) was used. To calculate PLVs, first, instantaneous phase of the $\theta$ oscillations was calculated via Hilbert transform and was denoted as $\phi_{\mathrm{Fr}}$ $(t)$ and $\phi_{\text {vis }}(t)$ for the frontal and visual areas, respectively. Second, PLV was calculated in each stimulation period excluding the first $0.5 \mathrm{~s}$ (i.e., from $t=0.5$ to $t=4$ ) as follows,

$$
\operatorname{PLV}=\frac{1}{T}\left|\sum_{t=0}^{T-1} \exp (i \Delta \phi(t))\right|
$$

where $\Delta \phi(t)=\phi_{\mathrm{Fr}}(t)-\phi_{\mathrm{Vis}}(t)$. PLV ranges from 0 (i.e., absence of phase locking) and 1 (i.e., perfect phase locking).

A cross-correlation function (CCF) $\rho_{X, Y}(\Delta t)$ of visual and frontal $\theta$ oscillations, $X(t)$ and $Y(t)$, respectively, was calculated in each trial excluding the first $0.5 \mathrm{~s}$ (i.e., from $t=0.5$ to $t=4$ ), according to 


$$
\rho_{X, Y}(\Delta t)=\frac{1}{\sigma_{X} \sigma_{Y}} \cdot \frac{1}{T} \sum_{t=0}^{T-1}\left(X(t)-\mu_{X}\right)\left(Y(\mathrm{t}+\Delta t)-\mu_{Y}\right),(7)
$$

in which $\mu$ and $\sigma$ indicate the mean and standard deviation. The time delay, $\tau$, was obtained from;

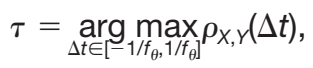

where arg max indicates the arguments of the maxima (i.e., the points that the CCFs are maximized). Positive values of $\tau$ indicate the temporal lead of visual $\theta$ over frontal $\theta$ and vice versa. The resolution of $\tau$ was $0.5 \mathrm{~ms}$ (2000-Hz sampling).

\section{Statistical analysis}

Non-parametric versions of $t$ test (Wilcoxon signed-rank and rank-sum test), ANOVA (Kruskal-Wallis test), and circular-median test using CircStat Toolbox (Berens, 2009) were performed ( $\alpha=.05$, two-tailed). For correlation analysis, Pearson's method was used with the same $\alpha$ level. Sample size was not previously estimated for the experiments, and blinding or randomization was not needed in this work, as the design was decided by subjects' performance.

\section{Results}

\section{Changes of behavioral performance during Go/No- Go task}

We trained mice $(n=5)$ to associate rightward motion $(\mathrm{S}+)$ with reward (water) and downward motion (S-) with punishment (air puff; Fig. 1A,B). The movement of visual stimulus was ceased at licking (i.e., black blank screen), as exemplified in Figure 1C. After training (for details, see Materials and Methods), we monitored the performance of the mice during the Go/No-Go test sessions for 10 successive days. During the test days, overall discrimination performance measured by D-prime ( $\mathrm{M} \pm \mathrm{SE}, d^{\prime}=$ $0.475 \pm 0.08$ ) remained above the chance level (i.e., $d^{\prime}=$ 0 , Wilcoxon signed-rank test, $Z=5.845, p<0.001$; for details, see Extended Data Fig. 1-1). There was no statistically significant difference in the discrimination performance across the test days (Kruskal-Wallis test, $\chi^{2}=$ 1.179, $p=0.999$ ). Moreover, a paired comparison between performance of the first half ( $1-5 \mathrm{~d})$ versus the last half $(6-10 \mathrm{~d})$ did not yield any statistically significant difference (Wilcoxon signed-rank test, $Z=0.405, p=$ 0.686 ), indicating the absence of a learning effect (Extended Data Fig. 1-1A).

On the other hand, the animals showed clear changes in performance within a single session, probably due to the change of motivational factors (e.g., fatigue and quenched thirst; Extended Data Fig. 1-1B,C). The timedependent patterns of performance were analyzed by dividing the session into early, middle, and late phases. When we classified the trials by hit (licking to $S+$ ), CR (no licking to $S-$ ), Miss (no licking to $S+$ ), and false alarm (FA; licking to $S-$-; Fig. $1 D$, the ratios and numbers of classified trials), the rate of correct answers did not show any time dependency: sum of hit and CR ratios was $57.1 \%, 59.9 \%$, and $57.4 \%$ in the early, middle, and late phases, respectively. Instead, the rates of licking decreased over time, shown through the sum of hit and FA ratios which was $64.7 \%, 51.6 \%$, and $37.1 \%$ in the early, middle, and late phases, respectively, possibly due to the decreased motivation for the task (for details, see Extended Data Fig. 1-1).

\section{Prominent $\theta$ rhythms in frontal and visual cortices during $\mathbf{C R}$}

All analyses of EEG data were focused on the time period from $0.5 \mathrm{~s}$ after stimulus onset until the cessation of stimulus (4 s after stimulus onset) during CR trials $(n=2411$ from five mice). As expected, our results showed that during the stimulus period of CR trials, $\theta$-band activities increased in both the frontal and visual cortices (Fig. 1E,F). Elicitation of $\gamma$ activities in the low $\gamma$ band (around $40 \mathrm{~Hz}$ ) was also observed in the visual cortex (Fig. 1E,F). The peak frequency of baseline-corrected induced $\theta$ amplitudes was $7.62 \mathrm{~Hz}$ $(F W H M=6.26 \mathrm{~Hz})$ and $6.64 \mathrm{~Hz}(\mathrm{FWHM}=4.96 \mathrm{~Hz})$ in the frontal and visual cortices, respectively. The stimulus-locked and response-locked spectrograms in all trials are summarized in Extended Data Figure 1-2. Interestingly, although the behavior was the same (i.e., absence of licking response), Miss trials did not display such prominent $\theta$-band activity (Extended Data Fig. 1-2).

\section{Assessment of attentional state using short-term task performance}

Because of the nature of the Go/No-Go task, some trial epochs without active engagement in the task (e.g., attentional lapse) could be counted as correct No-Go responses. To distinguish those by-accident $\mathrm{CR}$ trials from the intended, elaborate No-Go responses, we tracked the changes in attentional state within a single session. During a task, attentional states change and correspondingly, performance fluctuates over time (Clayton et al., 2015). In human and non-human primate studies, the short-term history of correctness (Liebe et al., 2012) or RT (Esterman et al., 2013) is often used to track the change of behavioral state. Similarly, we tracked the short-term proportion correct, $p_{c}$, by defining it as the ratio of correct trials (i.e., hit and $\mathrm{CR}$ ) across the 10 previous trials (see Materials and Methods for details). With a rough range of $2-5 \mathrm{~min}, p_{c}$ monitors the momentarily fluctuating, varying trial-to-trial, attentional state. The distribution of $p_{c}$ for all $\mathrm{CR}$ trials exhibited a positively shifted Gaussian function $(\mu=0.66$, $\sigma=0.23, R^{2}=0.98$; Fig. $2 A$ ) whose mean was above the chance level (i.e., $\mu=0.5$; one-sample $Z$ test, $Z=23.532$, $p<0.001$ ). Using the chance level as a threshold, we regarded the trial epochs as either attentive (i.e., $p_{c}$ above the chance level; $p_{c}>0.5$ ) or inattentive states (i.e., $p_{c} \leq$ $0.5)$. For CR trials, the lowest value of $p_{c}$ was 0.2 and the highest value was 1 , and attentive states were observed twice as frequently as inattentive states (1550 attentive states, 861 inattentive states). When we drew RT as a function of $p_{c}$ for licking cases (i.e., hit and FA), RT decreased in the case of hit trials (from $1.93 \mathrm{~s}$ for $p_{c}=$ $0.2-1.14 \mathrm{~s}$ for $p_{c}=1, Z=3.047, p=0.002$; Fig. $2 B$ ). On the other hand, RT in the case of FA trials did not show any dependence on $p_{c}$. In this way each trial was labeled with $p_{c}$, which successfully reflected the change of attentional state, for further analysis. 

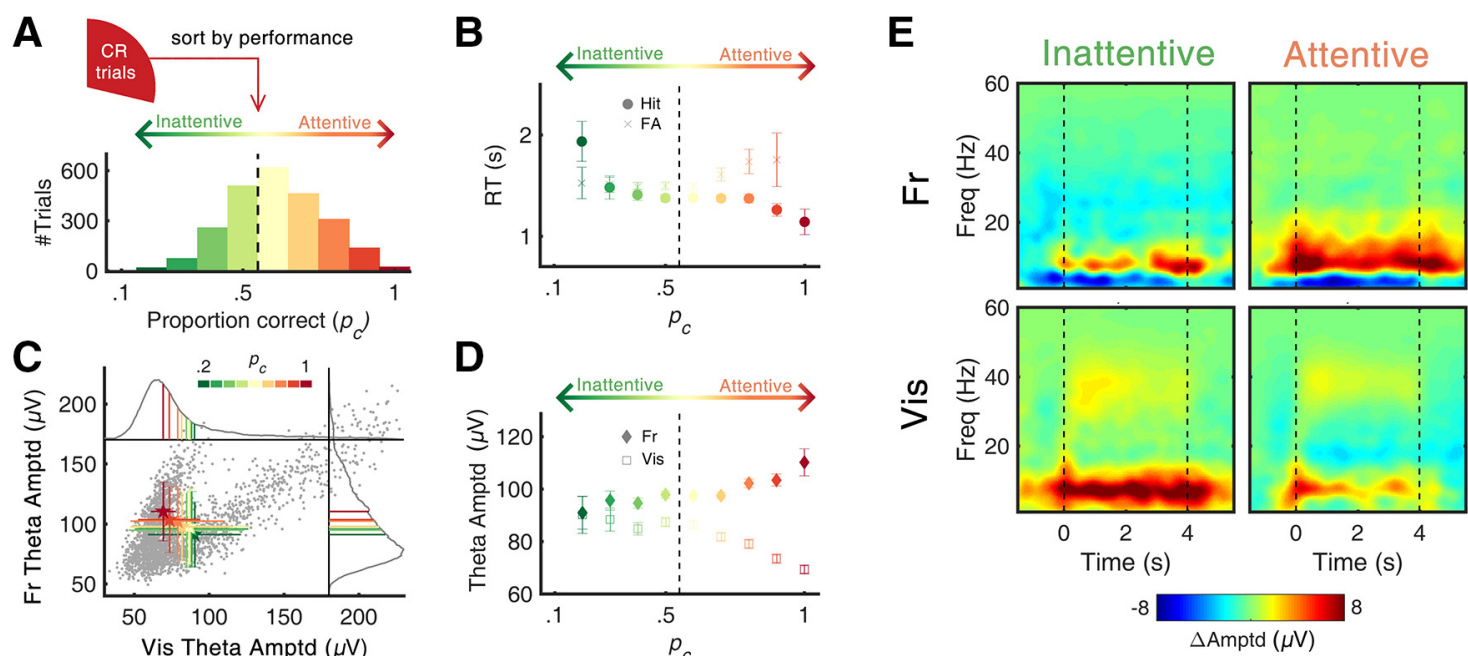

Figure 2. Task performance and $\theta$ amplitude. $\boldsymbol{A}$, Distribution of $p_{c}$ (proportion correct) in all CR trials $(n=2411) . p_{c}$ was calculated across 10 previous trials. With a threshold of chance level $\left(p_{c}=0.5\right)$, each trial was classified as either attentive $\left(p_{c}>0.5\right)$ or inattentive $\left(p_{c} \leq 0.5\right)$ state. $\boldsymbol{B}$, Change of mean RT as function of $p_{c}$. The RT of hit trials decreased as $p_{c}$ increased (Pearson's $r=-0.051, p<0.05$ ), suggesting the change of attentional level within the hit trial epochs. $\boldsymbol{C}$, Scatterplot of frontal/visual $\theta$ amplitude with color coding of $p_{c}$. Each gray dot represents the observed $\theta$ amplitude value of a single CR trial. Gray solid lines indicate the probability density function of each channel. Along with the changes of $p_{c}$, the mean amplitudes of frontal and visual $\theta$ showed a negative relationship. $\boldsymbol{D}$, Mean amplitude of frontal/visual $\theta$ as function of $p_{c}$. The linear correlation between $\theta$ amplitude and $p_{c}$ was positive in the frontal $(r=0.076, p<0.001)$ and negative in the visual $(r=-0.099, p<0.001)$, suggesting their distinct behavioral correlates. $\boldsymbol{E}$, Time-frequency representation of oscillatory amplitudes highlighting state-dependent and region-dependent change of $\theta$ amplitudes. Black dashed lines indicate the start/end of the visual stimuli. Error bars represent $\pm 1 \mathrm{SD}(\boldsymbol{C})$ and $S E(\boldsymbol{B}, \boldsymbol{D})$ of the means. $p_{c}$ : probability correct. The changes in power spectral density and its peak frequency were calculated for different values of $p_{c}$ (Extended Data Fig. 2-1).

\section{Opposite effects of attentional state on frontal and visual $\theta$ amplitudes}

The primary aim of the experiment was to articulate the ongoing $\theta$-activity signatures with respect to attention, which were reported to act in an opposite way in the frontal (Clayton et al., 2015) and visual (Spyropoulos et al., 2018) cortices. Thus, we were interested in the differences in ongoing $\theta$ activities between two attentional states. The scatterplot in Figure $2 \mathrm{C}$ shows a positive correlation between frontal and visual $\theta$ amplitudes. On the other hand, the mean of each $p_{c}$ presented as a colored error bar shows a negative correlation, with a movement in their distribution from the lower-right to the upper-left corner as $p_{c}$ is increased. This opposite behavior of frontal and visual $\theta$ is manifested in the plot of $\theta$ amplitudes as a function of $p_{c}$ (Fig. 2D). In the frontal cortex, the correlation between $\theta$ amplitude and $p_{c}$ was positive, reaching its maximum at $p_{c}=1$ (Pearson's $r=+0.08, p<0.001$ ). In contrast, the relationship was negative for visual $\theta$, reaching its minimum at $p_{c}=1$ (Pearson's $r=-0.10, p<$ $0.001)$. The average $\theta$ amplitudes for attentive versus inattentive, analyzed in the time-frequency domain, demonstrates the opposite effect of attention on the frontal and visual $\theta$ amplitudes (Fig. 2E). In the frontal cortex, $\theta$ amplitude in attentive states was significantly larger compared to that in inattentive states $(96.5 \pm 0.8 \mu \mathrm{V}$ in inattentive, $99.1 \pm 0.7 \mu \mathrm{V}$ in attentive; Wilcoxon rank-sum test, $Z=3.189, p<0.001)$. On the other hand, in the visual cortex, the amplitude of $\theta$ in attentive states was significantly smaller compared to that in inattentive states $(86.7 \pm 1.0 \mu \mathrm{V}$ in inattentive, $82.2 \pm 0.9 \mu \mathrm{V}$ in attentive; Wilcoxon rank-sum test, $Z=-2.582, p<0.001$ ). These contrasting correlations suggest that frontal and visual $\theta$ activities might have different functional roles during the engagement of cognitive processes required for the No-Go response, raising a question about the neurologic signature of sustained attention.

\section{Distinct pattern of phase-amplitude coupling with fast oscillations}

To further investigate the role of $\theta$ in each region, we performed the phase-amplitude CFC analysis, whose characteristics are suggested to reflect the architecture and function of underlying neuronal circuits (Hyafil et al., 2015). The amplitude maps of fast oscillations plotted with respect to $\theta$ phase revealed strong but different CFC patterns across different $p_{c}$ values and regions (Fig. $3 A$ ). For example, in the high-performance epochs with higher $p_{c}$, the amplitude of fast oscillations was strongly modulated by the phase of $\theta$ oscillation in the frontal cortex compared to in the low-performance epochs. To analyze the effect of behavioral state on CFC, we calculated the MI (Tort et al., 2010) as an indicator of CFC strength. As depicted in Figure $3 B$, the $\mathrm{MI}$ in the frontal cortex showed frequency-specific modulation of behavioral state, while the visual cortex did not show such modulation. Between $\theta$ and low $\gamma(20-40 \mathrm{~Hz})$, the attentional enhancement in the frontal CFC was statistically significant (inattentive: $6.1 \times 10^{-4} \pm 0.1 \times 10^{-4}$, attentive: $6.6 \times 10^{-4} \pm 0.1 \times$ 10 ${ }^{-4}$; Wilcoxon rank-sum test, $Z=2.187, p=0.029$; Fig. $3 C$ ). For the faster frequency band (high $\gamma, 80-160 \mathrm{~Hz}$ ) in the frontal cortex, attentional reduction was observed (inattentive: $7.4 \times 10^{-4} \pm 0.2 \times 10^{-4}$, attentive: $7.0 \times$ $10^{-4} \pm 0.1 \times 10^{-4}$; Wilcoxon rank-sum test, $Z=-2.546$, 
A

$$
\text { A }
$$
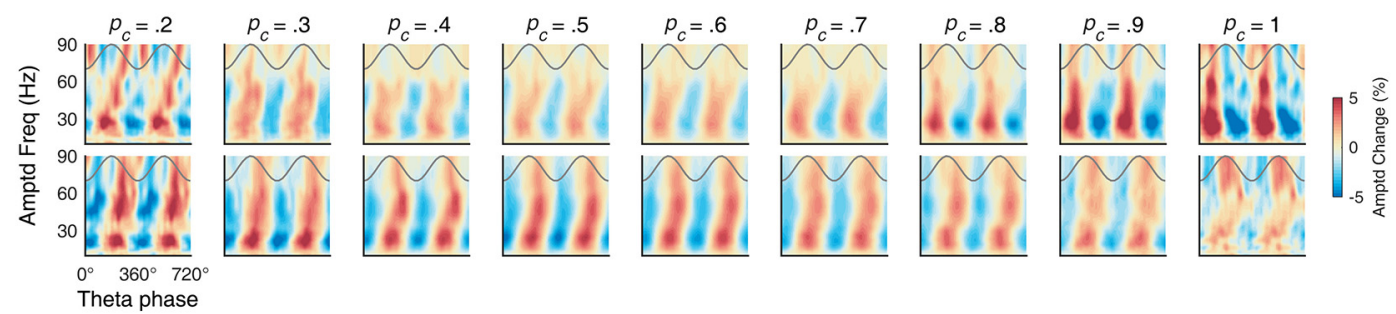

\section{B}
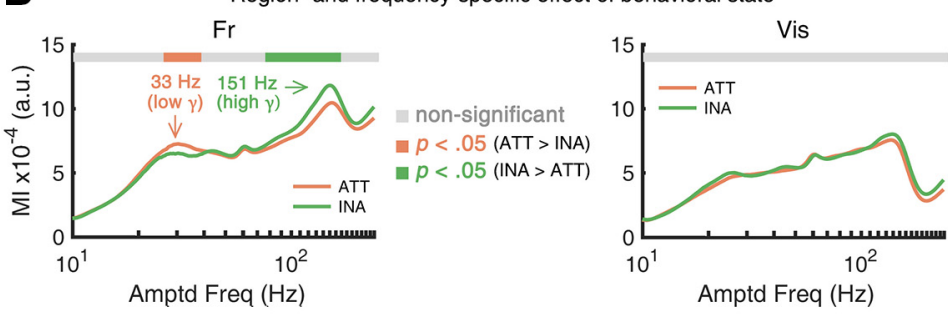

C
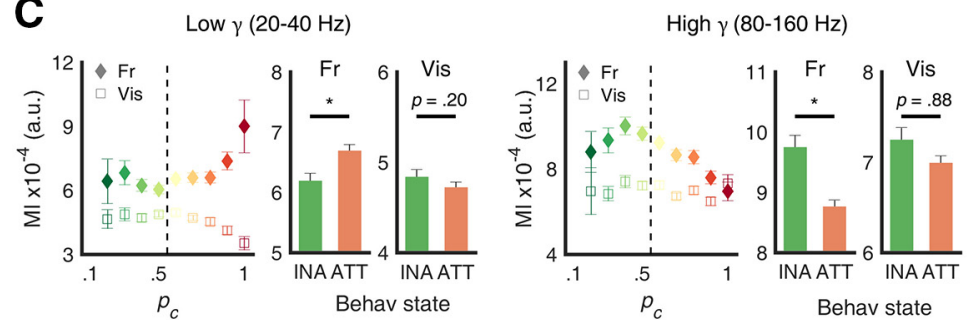

D Regional difference (Grand-averaged)

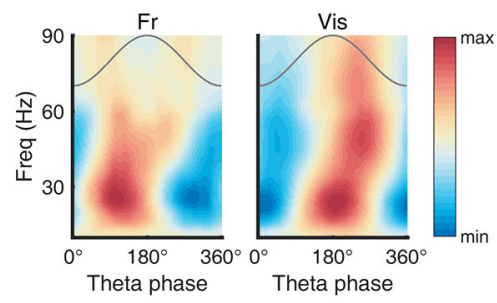

$\mathbf{E}$
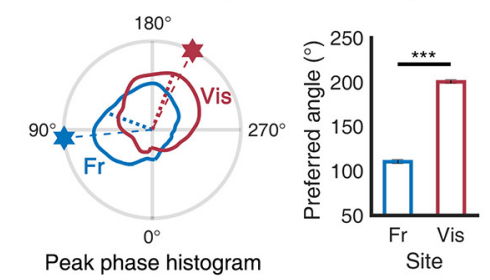

Figure 3. Task performance and $\theta-\gamma$ phase-amplitude coupling. $\boldsymbol{A}$, Color-coded fast-frequency amplitudes as function of $\theta$ phase in frontal (top) and visual (bottom) and as function of $p_{c}$ (left to right). $\boldsymbol{B}, \boldsymbol{C}, \mathrm{Ml}$ for local $\theta-\gamma$ coupling as function of $p_{c}$ (left: frontal, right: visual). In the frontal, the effect of attention on CFC was frequency-specific (increased CFC with low $\gamma$, decreased CFC with high $\gamma$ ). The attentional modulation of the visual coupling was not significant. The dependency of CFC on $\theta$ power or attentional state or their interaction was further analyzed using a general linear model, showing both $\theta$ power and its interaction with attentional state are significant independent parameters for CFC values (Extended Data Fig. 3-1). D, Grand-averaged amplitudes of fast oscillations as function of $\theta$ phase, showing noticeable regional difference of preferred $\theta$ phase (left: frontal, right: visual). $\boldsymbol{E}$, Trial histogram of preferred $\theta$ phase of $20-$ to $40-\mathrm{Hz}$ oscillations (left). Hexagons and dotted lines denote the peak and mean of each polar histogram, respectively. Mean preferred angle of each area (right). The distances between preferred angles over sites were both significant. Error bars represent \pm 1 SEM; $* p<0.05, * * * p<0.001$. INA: inattentive, ATT: attentive.

$p=0.011)$. However, in the visual cortex, the attentional effect on CFC was not statistically significant for either low $\gamma(Z=-1.443, p=0.149)$ or high $\gamma(Z=1.121, p=$ 0.262 ). It should be taken into consideration that the CFC changed due to changing $\theta$ amplitudes, rather than behavioral performance, as previous studies have shown that CFC can be either dependent on the amplitude of ongoing $\theta$ oscillations (Canolty et al., 2006; Tort et al., 2008) or independent from it (Kendrick et al., 2011, their Fig. S9). To disentangle this possible confounding factor, an additional single-trial GLM analysis was performed. The results suggest that both factors, $\theta$ amplitude and attentional state, contribute to the change of CFC (Extended Data Fig. 3-1).

These region-specific behaviors of CFC were further analyzed by investigating the $\theta$ phase at which $\gamma$ amplitudes peaked (i.e., peak preference). This was done to check that the two $\theta$ oscillations are qualitatively distinct, as previous studies have shown that such peak phase difference implies differences in the underlying circuit motif (Voloh and Womelsdorf, 2016, their Fig. 4). When these phase angles were pooled separately for each region, their distributions differed (Fig. 3D, polar plots). In the frontal cortex, the preferred $\theta$ phase (i.e., a phase bin of $\theta$ where maximum low $\gamma$ amplitude was detected) was observed in the middle of the rising phase $\left(109.7 \pm 2.0^{\circ}\right)$, whereas in the visual cortex, the preferred $\theta$ phase was observed near the start of the falling phase $\left(191.1 \pm 1.7^{\circ}\right)$. The distributions of the preferred $\theta$ phase of low $\gamma$ were statistically different for the frontal cortex and the visual cortex (circular Kruskal-Wallis test, $\chi^{2}=251.140, p<$ 0.001 ). The angle distance of the preferred $\theta$ between them was $81.4^{\circ}$, corresponding to $32.3 \mathrm{~ms}$ at $7 \mathrm{~Hz}$. In addition to the results from distinct behavioral modulation (Fig. $2 C-E$ ), the result of our CFC analysis presented here provides more reliable evidence that the $\theta$ rhythms of each area have distinct functional roles in the cortical microcircuit.

\section{Enhanced fronto-visual functional connectivity during attentive state}

Phase synchrony analysis allows the examination of signals that are cooperative between brain areas. To investigate the dynamic interplay of the fronto-visual $\theta$ network as attention fluctuates, we first examined the phase relationship between two $\theta$ rhythms using a joint phase 
A

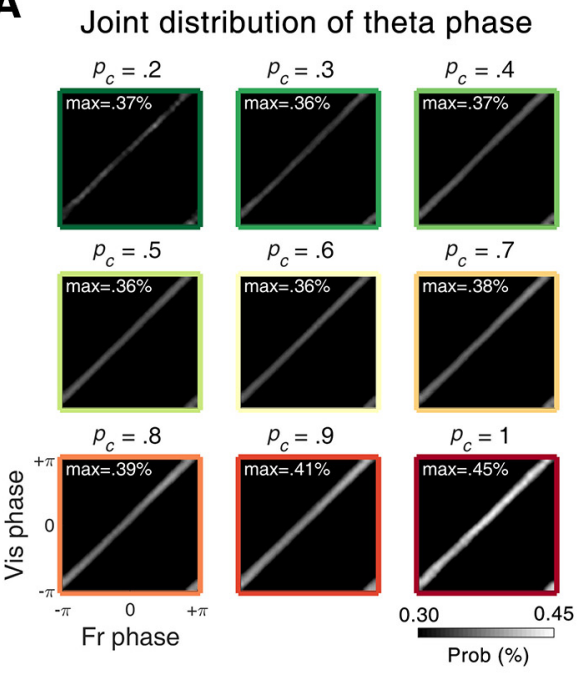

B

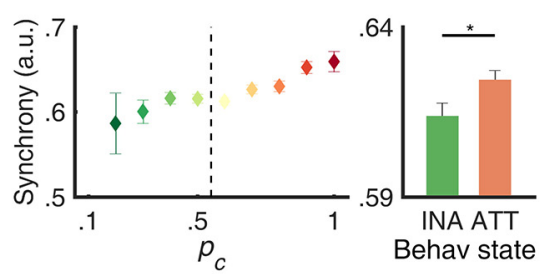

C

\section{Fr-Vis Cross-correlation}

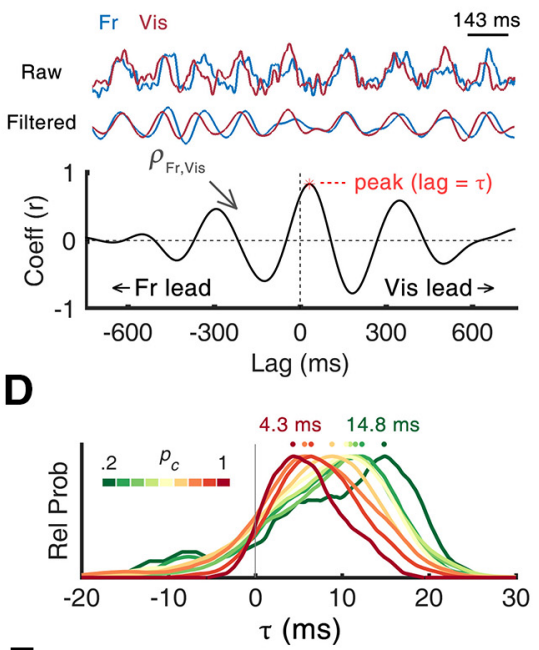

E

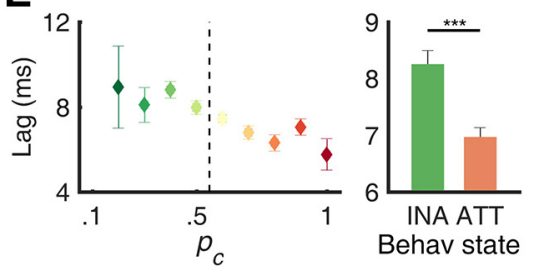

Figure 4. Synchrony and lags of $\theta$ oscillations. A, Two-dimensional representation of joint phase histogram of frontal and visual $\theta$ oscillations as function of $p_{c}$. The white color of each histogram indicates the higher probability of colocalization of the phase of two oscillations, suggesting a higher level of synchrony between two oscillations. The inset text indicates the maximum value of colocalization probability in each $p_{c}$ bin. $\boldsymbol{B}$, Mean fronto-visual phase synchrony calculated by PLV as function of $p_{c}$. The phase synchrony was higher for the attentive state than the inattentive state. Extended Data Figure 4-1A illustrates the phase-locking behaviors of two rhythms appeared as a transient locking. The phase synchrony is its maximal at the moment of frequency locking of two $\theta$ rhythms (Extended Data Fig. 4-1B), which is also dependent on the attentional state (Extended Data Fig. 4-1C). C, Cross-correlation analysis of example EEG epoch. Top, To calculate temporal lag $(\tau)$, raw time traces of EEG data from two channels were bandpass filtered in the $\theta$ band. Bottom, After filtering, the CCF was obtained and $\tau$ was defined as a temporal lag where the correlation coefficient reaches its maximum. Positive and negative values of $\tau$ indicate a lead of the visual and frontal $\theta$, respectively. $\boldsymbol{D}$, Trial histograms of $\tau$ between frontal and visual $\theta$. The dots denote the peak of each histogram. Color-coded texts indicate the peak position from the lowest $p_{c}$ (green) and to the highest $p_{c}$ (red) bin. $\boldsymbol{E}$, Mean $\tau$ as function of $p_{c}$. The $\tau$ was smaller in the attentive state than in the inattentive state. Error bars represent \pm 1 SEM; $* p<0.05, * * * p<0.001$. INA: inattentive, ATT: attentive.

histogram (see Materials and Methods for details). Figure $4 A$ highlights the systematic change of the instantaneous phase relationship as a function of $p_{c}$. In general, the joint phase of frontal and visual $\theta$ showed the stronger colocalization along the diagonal axis regardless of behavioral state, which indicates their intimate phase relationship. Furthermore, the colocalization along the diagonal axis became more robust as $p_{c}$ increased, suggesting a higher level of phase synchrony between the two $\theta$ rhythms. The PLV (Lachaux et al., 1999), an amplitude-independent parameter, elucidates the brain state-dependent synchrony between frontal and visual $\theta$ oscillations (Fig. 4B). Compared to the inattentive state, the PLV was significantly higher in the attentive state (PLV $=0.614 \pm .004$ in inattentive, $0.624 \pm .003$ in attentive; Wilcoxon rank-sum test, $Z=-2.313, p=0.021$ ). Our result of a fronto-visual $\theta$ synchrony associated with task performance is consistent with a previous finding in a non-human primate study (Liebe et al., 2012). While an opposite relationship between frontal and visual $\theta$ amplitudes was found in asso- ciation with the behavioral state, a stronger fronto-visual long-range functional network was established, especially when frontal $\theta$ was relatively increased and when visual $\theta$ was relatively suppressed.

The asymmetrical joint phase distribution (Fig. 4A, bottom-right corner of each joint phase histogram) raised the possibility of a driver-responder relationship between frontal and visual $\theta$. Therefore, we investigated the time lag, $\tau$, of frontal $\theta$ with respect to visual $\theta$ by finding the peaks at the CCFs of the two $\theta$ rhythms. Figure $3 C$ illustrates an example CCF of frontal and visual $\theta$ rhythms. $\tau$ was obtained at each trial and the distributions of $\tau$ were plotted for different $p_{c}$ values (Fig. 4D). Among all trials, a majority of the trials $(84.61 \%)$ had positive $\tau$, indicating that visual $\theta$ predominantly precedes frontal $\theta$. In addition, as $p_{c}$ increased, the distribution of $\tau$ shifted to shorter phase lag. The peak $\tau$ in Figure $4 D$ systematically decreased as the state changed from inattentive to attentive, with values of 14.83 and $4.33 \mathrm{~ms}$ at $p_{c}$ of 0.2 and 1 , respectively. $\tau$ was $8.25 \pm 0.24$ and $6.97 \pm 0.17 \mathrm{~ms}$ for 
inattentive and attentive states, respectively (Wilcoxon rank-sum test, $Z=4.832, p<0.001$ ). In addition, we found that the peak frequency of the cross-spectral density function was significantly higher for the attentive state $(5.84 \pm 0.03 \mathrm{~Hz})$ compared to the inattentive state $(5.76 \pm$ $0.04 \mathrm{~Hz}$; Wilcoxon rank-sum test, $Z=2.052, p=0.040$; Extended Data Fig. 2-1). The increase of synchrony and shift to shorter phase lag during the attentive state suggests the contribution of $\theta$ oscillations to task engagement and sustained attention via their long-range functional network.

It may seem odd that we calculated the phase synchrony after demonstrating that the two oscillators are distinct in peak frequency, since two oscillations must have transient periods of same frequency for phase locking to occur. Indeed, when we calculated synchrony for various instantaneous $\theta$ frequency, we found there were times during which the two $\theta$ rhythms oscillated in sync (Extended Data Fig. 4-1). Frequency difference was low when high phase synchrony was observed, and the probability of converging frequency difference (to 0 ) was found to be modulated by attention, which explains why PLV was higher during the attentive state.

\section{Discussion}

We found that the frontal and visual $\theta$ induced by the task had functionally distinct features. Whereas the frontal $\theta$ amplitude was positively correlated with behavioral performance, the visual $\theta$ amplitude was negatively correlated. In the frequency domain, the peaks of the two $\theta$ oscillations differed by almost $1 \mathrm{~Hz}$, and their phases modulated fast cortical oscillations in different ways. Nonetheless, the frontal-visual $\theta$ synchrony increased as a function of performance despite the weakened amplitude of visual $\theta$, implying a critical role of phase-locked oscillations in successful task engagement. It is unlikely that frontal and visual $\theta$ have common driving mechanisms; instead, it is more likely that the two $\theta$ have independent origins but are weakly coupled to each other. We should note that there is a distinction between phase locking (i.e., coupled oscillation with a constant phase difference) and phase trapping (i.e., un-locked oscillation with the same frequency) in nonlinear oscillator systems (Aronson et al., 1990). Indeed, the frontal and visual $\theta$ in our observation showed a phase transition from a stable phase-trapping system to a non-symmetric phase-locking system as the attention level increased. Although the differences in the values were small, we observed that there were abrupt changes in the interaction parameters (e.g., max in joint phase histogram, synchrony, and lag in Fig. 4) near $p_{c}=$ 0.8. Furthermore, the behavioral parameters (i.e., RT in Fig. $2 B$ ) and the neural dynamics parameters (i.e., $\theta$ amplitude in Fig. 2D; $\theta-\gamma$ coupling in Fig. $3 A, C)$ presented their characteristic features near $p_{c}=0.8$. It is highly possible that the $\theta$ oscillations changed their dynamic trajectory at the highly task-engaged brain state in their phase portrait; however, determining whether this transition contributed to sustained attention or vice versa was beyond the scope of this study.
Our phase dynamics analysis showed that visual $\theta$ led frontal $\theta$ throughout the recording sessions. At the same time, frontal, not visual, $\theta$ increased its amplitude at higher $p_{c}$ accompanied by a reduction in its phase delay to visual $\theta$. This confounding behavior at higher $p_{c}$ could be an influence of top-down directed frontal $\theta$ for cognitive control such as motor inhibition (Cavanagh and Frank, 2014) or phase resetting of $\theta$ oscillations by a stimulus in a highly task-engaged brain state (Rizzuto et al., 2003). In terms of the Communication through Coherence theory (Fries, 2015), the tight interplay of two oscillations renders neuronal communication more effective, precise, and selective. It is possible that a new common source arose around $p_{c}=0.8$ to enhance their synchrony and that the newly adjusted phase lag was simply differential conduction delay. However, the different patterns of $\theta-\gamma$ coupling suggest this was not the case. Recently, Zhang et al. (2018) reported that human cortical oscillations were regionally distinctive and had the properties of weakly coupled oscillations rather than those of oscillations driven by a common source or feedback loop. Although attentional modulation was not included in their study, it was consistent with our observations, in that the visual rhythm was faster than the frontal rhythm by $\sim 1 \mathrm{~Hz}$ and propagated to the anterior cortex. However, there was a mismatch in the propagation delay in the human visual to frontal cortex, which was tenfold slower compared to our observation in mice or in monkeys (Gregoriou et al., 2009). The leading of visual $\theta$ might reflect the bottom-up relay of sensory information, and the systematic reduction of the delay is functionally relevant to its purpose of faster relaying of sensory information. The delay is interpreted as a designated temporal shift for the effective relay of information, transmitting a presynaptic spike at the peak depolarization phases of postsynaptic neurons (Nowak and Bullier, 1997; Gregoriou et al., 2009). Metaphorically speaking, to selectively improve the traffic in one direction, it may be optimal for the green lights of successive crossways to be slightly delayed considering the amount of time it takes to drive through, rather than operating with zero-lag synchrony.

Then what is the generation mechanism of frontal $\theta$ observed here? Considering that our task requires an effort to withhold impulsive actions, frontal $\theta$ might originate from the interaction of the cortico-basal ganglia circuit (i.e., anteriorcingulate/medial-frontal and subthalamic nucleus) related to the conflict-related decision process (Cavanagh et al., 2011; Cavanagh and Frank, 2014; Cohen, 2014). Or the frontal $\theta$ may reflect communication between hippocampal structures and the frontal cortex (Jones and Wilson, 2005; Siapas et al., 2005), as the task required the subjects to maintain visuospatial information (i.e., direction of motion). Regarding the role of frontal $\theta$ oscillations in sustained attention, a large and consistent body of literature has discussed the topdown role of frontal $\theta$ in cognitive control (Cavanagh and Frank, 2014) and sustained attention (Clayton et al., 2015). All these works included task-engaged mental processes (e.g., keeping one's goal in mind, overriding the prepotent response, monitoring the goal-related sensory cues). Our results provide further evidence for this top-down role of 
frontal $\theta$ oscillations, as we found that frontal $\theta$ increased during sustained attention in mice. Importantly, phase synchrony between frontal and visual $\theta$ increased in highperformance trials in a critical manner. Thus, frontal $\theta$ appears to be behaviorally relevant as its top-down control mode. It should be noted that the substantial work conducted in the frontal cortex has found $\gamma$ oscillations to play an important role in pro-cognitive functions (Cho et al., 2015; Kim et al., 2016). On the neuronal spike levels, the spiking activities of the frontal cortex in the high- $\gamma$ band were locked to $\theta$ oscillations in the frontal and posterior parietal cortices during sustained attention (Sellers et al., 2016). In our study, we found effective modulation of low $\gamma$, but not high $\gamma$, by frontal $\theta$ using CFC analysis. Continued work with neuronal recording or optogenetic interrogation will be needed to clarify the specific role of frontal $\theta$ on neuronal activities during cognitive processing.

The counteracting behavior of visual $\theta$ compared to frontal $\theta$ was counterintuitive, but it was consistently and significantly observed across animals and days. This phenomenon of "attentional reduction" in visual $\theta$ is in agreement with recent findings in monkeys (van Kerkoerle et al., 2014; Spyropoulos et al., 2018) and ferrets (Stitt et al., 2018). In humans, it has been reported that $\alpha$ activities $(8-12 \mathrm{~Hz})$ in the occipital cortex reflect functional inhibition of task-irrelevant cortical areas (Jensen and Mazaheri, 2010). To the best of our knowledge, wakeful relaxation related $\alpha$ activities have not been reported in mice unlike other cross-species frequency-preserved rhythms (Buzsáki et al., 2013). In addition, compared to baseline activity, visual $\theta$ in our study increased during stimulus presentation (Fig. 2E), whereas $\alpha$ usually decreases (Fries et al., 2008), which is similar to the finding of Spyropoulos et al. (2018). Therefore, it seems more plausible to regard the oscillatory activities observed in this study as visual $\theta$ rather than occipital $\alpha$. However, the functional significance of such attentional reduction in the cortical microcircuit remains unknown.

What is the role of $\theta$ rhythmicity in the visual system? Recently, it has been suggested that the $\theta$ rhythmicity in the perceptual domain reflects the periodic sampling process of the sensory system (i.e., rhythmic sampling; VanRullen, 2016, 2018). In this framework, attention periodically fluctuates around the $\theta$ band to sample sensory stimuli, resulting in $\theta$-rhythmic fluctuations of relevant neural activities and behavioral responses (Busch and VanRullen, 2010; Fiebelkorn et al., 2018; Kienitz et al., 2018). Still, the suppression of visual $\theta$ by attention is not explained, particularly when we consider that $\theta$ rhythms reflect the attentional sampling process. According to Spyropoulos et al., the attentional reduction of visual $\theta$ was suggested to reflect "more continuous processing" of attended stimuli, and a shift to the faster rhythm was predicted (Spyropoulos et al., 2018). Moreover, Harris et al., reported that the phase modulation of visual $\theta$ on visual detection performance was more effective for a non-attended than attended target in humans (Harris et al., 2018). These results suggest that visual $\theta$ might be in charge of processing outside the attentional spotlight rather than involved in neural processing in the focal attentional field.
While a growing number of studies have revealed the modulation effects of visual $\theta$ on neural excitability, the neural basis for the generation of visual $\theta$ remains unknown (VanRullen, 2018). Recently, a predominant feedforward directionality of visual $\theta$ was found in monkeys, from lower to higher levels of the visual cortex, and to the temporal cortex (Spyropoulos et al., 2018). In addition, a colocalization of stimulus-induced $\gamma$ oscillations and visual $\theta$ oscillations was revealed (van Kerkoerle et al., 2014). Considering that the feedforward directionality of $\gamma$ oscillations in the primary sensory cortex is relatively well known, it is believed that visual $\theta$ reflects bottom-up sensory processing rather than top-down influences from higherorder visual areas. Moreover, simultaneous recording of the visual thalamic area and the posterior-parietal cortex in the ferret brain also demonstrated a strong feedforward directionality in the $\theta$ frequency band (Stitt et al., 2018), but it is not clear whether it is generated from the thalamo-cortical network or instead is locally driven.

If visual $\theta$ propagates in a feedforward direction, how can it be modulated by top-down processes such as attention? One recent study using ferret thalamus recordings suggests an answer to this question. It found that spiking activities in the pulvinar are modulated by attention, and these neurons signal in the $\theta$ frequency band during sustained attention ( $\mathrm{Yu}$ et al., 2018). This area exhibits bidirectional anatomic connections with higher-order visual cortices (Jones, 2001), and even with the prefrontal cortex (Romanski et al., 1997). As there has also been a report that unit activities in the primate visual area V4 are modulated by the external $\theta$ driver in the prefrontal cortex (Liebe et al., 2012), it seems highly plausible that the top-down modulation of $\theta$ is generated via a cortico-thalamic input, which in turn leads to a change of thalamo-cortical connectivity. Indeed, attentional modulation of neuronal activities in the visual cortices has attracted a wide range of interest among cognitive scientists. Considering the genetic techniques uniquely applicable to the mouse model, future studies may take advantage of mouse models to understand the functional architecture and underlying circuitry of visual $\theta$ rhythmicity in association with its attentional modulation.

\section{References}

Aronson DG, Ermentrout GB, Kopell N (1990) Amplitude response of coupled oscillators. Physica D 41:403-449.

Beatty J, Greenberg A, Deibler WP, O'Hanlon JF (1974) Operant control of occipital theta rhythm affects performance in a radar monitoring task. Science 183:871-873.

Benchenane K, Tiesinga PH, Battaglia FP (2011) Oscillations in the prefrontal cortex: a gateway to memory and attention. Curr Opin Neurobiol 21:475-485.

Berens P (2009) CircStat: a MATLAB toolbox for circular statistics. J Stat Softw 31:1-21.

Busch NA, VanRullen R (2010) Spontaneous EEG oscillations reveal periodic sampling of visual attention. Proc Natl Acad Sci USA 107:16048-16053.

Buzsáki G, Logothetis N, Singer W (2013) Scaling brain size, keeping timing: evolutionary preservation of brain rhythms. Neuron 80:751-764.

Canolty RT, Edwards E, Dalal SS, Soltani M, Nagarajan SS, Kirsch HE, Berger MS, Barbaro NM, Knight RT (2006) High gamma power is phase-locked to theta oscillations in human neocortex. Science 313:1626-1628. 
Cavanagh JF, Frank MJ (2014) Frontal theta as a mechanism for cognitive control. Trends Cogn Sci 18:414-421.

Cavanagh JF, Wiecki TV, Cohen MX, Figueroa CM, Samanta J, Sherman SJ, Frank MJ (2011) Subthalamic nucleus stimulation reverses mediofrontal influence over decision threshold. Nat Neurosci 14:1462-1467.

Cho KK, Hoch R, Lee AT, Patel T, Rubenstein JL, Sohal VS (2015) Gamma rhythms link prefrontal interneuron dysfunction with cognitive inflexibility in Dlx5/6(+/-) mice. Neuron 85:1332-1343.

Choi JH, Koch KP, Poppendieck W, Lee M, Shin HS (2010) High resolution electroencephalography in freely moving mice. J Neurophysiol 104:1825-1834.

Chun MM, Golomb JD, Turk-Browne NB (2011) A Taxonomy of external and internal attention. Annu Rev Psychol 62:73-101.

Clayton MS, Yeung N, Kadosh RC (2015) The roles of cortical oscillations in sustained attention. Trends Cogn Sci 19:188-195.

Cohen MX (2014) A neural microcircuit for cognitive conflict detection and signaling. Trends Neurosci 37:480-490.

Douglas RM, Neve A, Quittenbaum JP, Alam NM, Prusky GT (2006) Perception of visual motion coherence by rats and mice. Vision Res 46:2842-2847.

Esterman M, Noonan SK, Rosenberg M, Degutis J (2013) In the zone or zoning out? Tracking behavioral and neural fluctuations during sustained attention. Cereb Cortex 23:2712-2723.

Fiebelkorn IC, Pinsk MA, Kastner S (2018) A dynamic interplay within the frontoparietal network underlies rhythmic spatial attention. Neuron 99:842-853.

Fries $\mathrm{P}$ (2015) Rhythms for cognition: communication through coherence. Neuron 88:220-235.

Fries P, Womelsdorf T, Oostenveld R, Desimone R (2008) The effects of visual stimulation and selective visual attention on rhythmic neuronal synchronization in macaque area v4. J Neurosci 28: 4823-4835.

Gregoriou GG, Gotts SJ, Zhou H, Desimone R (2009) Highfrequency, long-range coupling between prefrontal and visual cortex during attention. Science 324:1207-1210.

Guo ZCV, Hires SA, Li N, O'Connor DH, Komiyama T, Ophir E, Huber D, Bonardi C, Morandell K, Gutnisky D, Peron S, Xu NL, Cox J, Svoboda K (2014) Procedures for behavioral experiments in headfixed mice. PLoS One 9:e88678.

Han HB, Hwang E, Lee S, Kim MS, Choi JH (2017) Gamma-band activities in mouse frontal and visual cortex induced by coherent dot motion. Sci Rep 7:43780.

Harris AM, Dux PE, Mattingley JB (2018) Detecting unattended stimuli depends on the phase of prestimulus neural oscillations. $J$ Neurosci 38:3092-3101.

Hyafil A, Giraud AL, Fontolan L, Gutkin B (2015) Neural crossfrequency coupling: connecting architectures, mechanisms, and functions. Trends Neurosci 38:725-740.

Jensen O, Mazaheri A (2010) Shaping functional architecture by oscillatory alpha activity: gating by inhibition. Front Hum Neurosci 4:186.

Jones EG (2001) The thalamic matrix and thalamocortical synchrony. Trends Neurosci 24:595-601.

Jones MW, Wilson MA (2005) Phase precession of medial prefrontal cortical activity relative to the hippocampal theta rhythm. Hippocampus 15:867-873.

Kendrick KM, Zhan Y, Fischer H, Nicol AU, Zhang X, Feng J (2011) Learning alters theta amplitude, theta-gamma coupling and neuronal synchronization in inferotemporal cortex. BMC Neurosci 12: 55.

Kienitz R, Schmiedt JT, Shapcott KA, Kouroupaki K, Saunders RC, Schmid MC (2018) Theta rhythmic aeuronal activity and reaction times arising from cortical receptive field interactions during distributed attention. Curr Biol 28:2377-2387.

Kim H, Ährlund-Richter S, Wang X, Deisseroth K, Carlén M (2016) Prefrontal parvalbumin neurons in control of attention. Cell 164: 208-218.
Lachaux JP, Rodriguez E, Martinerie J, Varela FJ (1999) Measuring phase synchrony in brain signals. Hum Brain Mapp 8:194-208.

Liebe S, Hoerzer GM, Logothetis NK, Rainer G (2012) Theta coupling between V4 and prefrontal cortex predicts visual short-term memory performance. Nat Neurosci 15:456-562.

Mazaheri A, Nieuwenhuis ILC, van Dijk H, Jensen O (2009) Prestimulus alpha and mu activity predicts failure to inhibit motor responses. Hum Brain Mapp 30:1791-1800.

Nowak LG, Bullier J (1997) The timing of information transfer in the visual system. In: Extrastriate cortex in primates, pp 205-241. New York: Springer.

Paxinos G, Franklin KB (2004) The mouse brain in stereotaxic coordinates. Houston: Gulf Professional Publishing.

Rizzuto DS, Madsen JR, Bromfield EB, Schulze-Bonhage A, Seelig $D$, Aschenbrenner-Scheibe R, Kahana MJ (2003) Reset of human neocortical oscillations during a working memory task. Proc Natl Acad Sci USA 100:7931-7936.

Romanski LM, Giguere M, Bates JF, Goldman-Rakic PS (1997) Topographic organization of medial pulvinar connections with the prefrontal cortex in the rhesus monkey. J Comp Neurol 379:313332.

Sauer JF, Strüber M, Bartos M (2015) Impaired fast-spiking interneuron function in a genetic mouse model of depression. Elife 4:e04979.

Sauseng P, Griesmayr B, Freunberger R, Klimesch W (2010) Control mechanisms in working memory: a possible function of EEG theta oscillations. Neurosci Biobehav R 34:1015-1022.

Sellers KK, Yu CX, Zhou ZC, Stitt I, Li YH, Radtke-Schuller S, Alagapan S, Fröhlich F (2016) Oscillatory dynamics in the frontoparietal attention network during sustained attention in the ferret. Cell Rep 16:2864-2874.

Siapas AG, Lubenov EV, Wilson MA (2005) Prefrontal phase locking to hippocampal theta oscillations. Neuron 46:141-151.

Spyropoulos G, Bosman CA, Fries P (2018) A theta rhythm in macaque visual cortex and its attentional modulation. Proc Natl Acad Sci USA 115:E5614-E5623.

Stitt I, Zhou ZC, Radtke-Schuller S, Fröhlich F (2018) Arousal dependent modulation of thalamo-cortical functional interaction. Nat Commun 9:2455.

Tort AB, Kramer MA, Thorn C, Gibson DJ, Kubota Y, Graybiel AM, Kopell NJ (2008) Dynamic cross-frequency couplings of local field potential oscillations in rat striatum and hippocampus during performance of a T-maze task. Proc Natl Acad Sci USA 105:2051720522.

Tort AB, Komorowski R, Eichenbaum H, Kopell N (2010) Measuring phase-amplitude coupling between neuronal oscillations of different frequencies. J Neurophysiol 104:1195-1210.

van Kerkoerle T, Self MW, Dagnino B, Gariel-Mathis MA, Poort J, van der Togt C, Roelfsema PR (2014) Alpha and gamma oscillations characterize feedback and feedforward processing in monkey visual cortex. Proc Natl Acad Sci USA 111:14332-14341.

VanRullen R (2016) Perceptual cycles. Trends Cogn Sci 20:723-735.

VanRullen R (2018) Attention cycles. Neuron 99:632-634.

Voloh B, Womelsdorf T (2016) A role of phase-resetting in coordinating large scale neural networks during attention and goaldirected behavior. Front Syst Neurosci 10:18.

von Stein A, Sarnthein J (2000) Different frequencies for different scales of cortical integration: from local gamma to long range alpha/theta synchronization. Int J Psychophysiol 38:301-313.

Vyazovskiy VV, Tobler I (2005) Theta activity in the waking EEG is a marker of sleep propensity in the rat. Brain Res 1050:64-71.

Yu C, Li Y, Stitt IM, Zhou ZC, Sellers KK, Frohlich F (2018) Theta oscillations organize spiking activity in higher-order visual thalamus during sustained attention. eNeuro 5: ENEURO.038417.2018.

Zhang HH, Watrous AJ, Patel A, Jacobs J (2018) Theta and alpha oscillations are traveling waves in the human neocortex. Neuron 98:1269-1281. 